\title{
Equilibrio Líquido Vapor en Sistemas Seudobinarios Alcohol - Agua - Electrolitos Fuertes a partir del Modelo UMIFAC - E
}

\author{
Jimmy Andrés López Jiménez* \\ Herlendy Lozada Salgado*
}

* Estudiante de Ingeniería Química - Departamento de Ingeniería Química - Universidad Macional de Colombia Manizales.

e-mail:jalopez@nevado.manizales.unal.edu.co e-mail:herlendyl@unalmzl.edu.co

Fecha de recepción: Septiembre 30 de 2004 Fecha de aprobación: Diciembre 17 de 2004

\section{RESUMEN}

El modelo de actividad UMIFAC-E, el cual es una expresión modificada de su versión original para sistemas no-electrolíticos, ha sido usado para calcular los equilibrios líquido vapor (ELV) de sistemas seudobinarios alcohol-agua-electrolitos fuertes. Se ha confirmado la precisión del modelo en estos sistemas y además se pudo comparar el efecto que tiene sobre el equilibrio de fases la adición de una sal, la cual en algunos casos posee la capacidad de trasladar el equilibrio en el sentido deseado: desplazamiento total del azeótropo en sistemas azeotrópicos y alejamiento de la curva de equilibrio de la línea de $45^{\circ}$, tanto en sistemas zeotrópicos como azeotrópicos. 
Palabras Clave: ELV en sistemas solvente-sal, modelo de actividad UMIFAC-E.

\section{ABSTRACT}

The UMIFAC-E activity model, which is a modified expression of their original version for nonelectrolytic systems, has been used to calculate the vapor liquid equilibrium (VLE) of alcohol-waterstrong electrolytes pseudo-binaries systems. The model's precision has been confirmed in these systems and could also compare the effect that has on the VLE the addition of a salt, which in some cases has the capacity to move the equilibrium in the wanted sense: total displacement of the azeotrope in azeotropic systems and estrangement of the curve of equilibrium of the line of $45^{\circ}$ both in zeotropics and azeotropic systems.

Key Words: VLE in solvent-salt systems; UMIFAC-E activity model.

\section{INTRODUCCIÓN}

Hay una gran variedad de importantes procesos industriales que involucran soluciones electrolíticas: tratamiento de aguas residuales, desalinización del agua de mar, destilación extractiva por efecto salino, cristalización normal o extractiva y procesos hidrometalúrgicos, son algunos ejemplos.

El modelado de las propiedades termodinámicas de soluciones electrolíticas se hace esencial para el diseño y simulación de estos procesos. Para algunos procesos, donde la concentración del electrolito está dentro de un rango diluido no se requiere del modelado de estas propiedades, mientras para rangos de concentraciones intermedias y altas, el modelado es de gran importancia.

Para un correcto modelado, las ecuaciones que representan las propiedades termodinámicas deben ser lo más exactas posibles con respecto a datos experimentales reportados en la literatura. Las deducciones de estas ecuaciones, en el caso de soluciones solvente-electrolito, son generalmente teórico-experimentales; en donde el aporte teórico proviene generalmente de la ley de fuerzas intermoleculares, en las cuales se tiene en cuenta la combinación de las fuerzas electrostáticas de largo alcance, donde generalmente se utiliza la teoría de DebyeHückel; con las interacciones de corto alcance; en la cual se manejan modelos de actividad convencionales como UMIFAC (Kikic et al., 1991), UMIQUAC (Macedo et al., 1990), MRTL (Chen et al., 1986) y Wilson (Zhao et al., 2000), que han sido modificados permanentemente para su uso en soluciones electrolíticas.

Sander, Fredenslud y Rasmussen (1986) presentaron un método para la corrección y predicción del efecto salino sobre el equilibrio líquido vapor. El modelo combina los términos de Debye-Hückel y la ecuación de UMIQUAC modificada con parámetros dependientes de la concentración. La suposición básica en el modelo de sander y en los modelos subsecuentes, es la de no tener en cuenta la asociación de aniones y cationes en el desarrollo de la teoría de Debye-Hückel, hecho fácilmente asimilable para el caso de solventes con constantes dieléctricas bajas, lo cual ocasiona la completa dilución del electrolito en el solvente. Además, estos autores establecieron como constantes los parámetros de Debye-Hückel: A y $b$, haciéndolos independientes de la temperatura del sistema y de la constante dieléctrica del solvente. Macedo, Skovborg y Rasmussen (1990) modificaron el término de Debye-Hückel usado por Sander para asegurar una correcta representación de las fuerzas electrostáticas de largo alcance. Dicha modificación requirió de una reestimación de los parámetros previamente publicados por Sander. Macedo et al. (1990) también mostraron que el modelo modificado tiene tanta exactitud como el modelo de Sander, igualmente extendieron el rango de aplicabilidad del modelo de Sander, debido a que incluyeron 
los parámetros de dos solventes y siete iones. Kikic, Fermeglia y Rasmussen (1991) modificaron el modelo propuesto por Sander: (1) el término de Debye-Hückel es calculado de acuerdo a la teoría de soluciones de McMillanMayer tal como lo describen Cardoso y O'Conell (1987) y (2) el término de UMIQUAC fue sustituido por el término UMIFAC. Kikic et al. (1991) estimaron los parámetros de interacción de UMIFAC entre iones y solventes, mientras que los parámetros de interacción entre los grupos de solventes son los reportados para sistemas noelectrolíticos (Abbott y Prausnitz, 1994).

\section{MODELO UNIFAC - E}

El modelo UMIFAC-E usado en este trabajo es identico al presentado por Kikic et al. (1991). En dicho modelo, el coeficiente de actividad de cualquiera de los solventes presentes en la fase líquida depende de las fuerzas intermoleculares de largo y corto alcance. Las fuerzas de largo alcance, que aparecen cuando las moléculas se encuentran a distancias en las que no hay sobreposición de sus nubes electrónicas, son calculadas a partir de la teoría de Debye-Hückel, bajo la suposición de que no existe asociación entre aniones y cationes en la fase líquida. Las fuerzas de corto alcance, también llamadas fuerzas de valencia o fuerzas químicas, son calculadas a partir del modelo convencional UMIFAC para sistemas no-electrolíticos. Los parámetros de interacción entre los grupos de iones y solventes usados en el modelo convencional de UMIFAC, fueron estimados y reportados por Kikic et al. (1991).

El coeficiente de actividad $\gamma_{n}$ del solvente $n$ en una mezcla líquida de solventes, es calculado como:

$1 n \gamma_{n}=1 n \gamma_{n}^{D-H}+1 n \gamma_{n}^{c}+1 n \gamma_{n}^{R}$

Donde $\gamma_{n}^{D-H}$ es el coeficiente de actividad de Debye-Hückel y $\gamma_{n}^{C}$ y $\gamma_{n}^{R}$ representan las contribuciones combinatoria y residual de UMIFAC.

\subsection{Coeficiente de actividad de Debye-Hückel:}

El parámetro de Debye-Hückel es calculado mediante la ecuación descrita por Macedo et al. (1990):

$1 \mathrm{n} \gamma_{n}^{\mathrm{D}-H}=\frac{2 \cdot \mathrm{A} \cdot \mathrm{M}_{n} \cdot \mathrm{d}_{5}}{\mathrm{D}^{3} \cdot \mathrm{d}_{n}}\left[1+\mathrm{D} \sqrt{I}-\frac{1}{1+\mathrm{b} \sqrt{I}}-21 \mathrm{n}(1+\mathrm{D} \sqrt{I})\right]$

Donde, $M_{n}$ es la masa molar del solvente $n, I$ es la fuerza iónica, $d_{n}$ es la densidad del solvente puro n. y $d_{s}$ es la densidad de la mezcla de solventes.

$d_{s}=\frac{M_{s}}{\sum_{n}^{\prime} \cdot M_{n} / d_{n}}$

$x_{n}^{\prime}$ es la fracción molar del solvente $n$ libre de sal, $M_{s}=\sum x_{n}^{\prime} . M_{n}$ es la masa molar de la mezcla líquida.

Las constantes de la ecuación de Debye-Hückel, al contrario de las usadas por Sander et al. (1986), se consideran una función directa de las propiedades de los solventes:

$A=1.327757 \times 10^{5} \cdot d_{s}^{1 / 3} /(\varepsilon \cdot T)^{3 / 2}$

$\mathrm{b}=6.359696 . \mathrm{d}_{\mathrm{s}}^{1 / 2} /(\varepsilon . T)^{1 / 2}$

Donde $\varepsilon$ es la constante dieléctrica de la mezcla de solventes, la cual es obtenida de los valores de los solventes puros $\varepsilon_{n}$ y la regla empírica de mezclado de Oster's, la cual para mezclas acuosas binarias (agua es el solvente 1) puede aproximarse a:

$\varepsilon=\varepsilon_{1}+\left[\left(\varepsilon_{2}-1\right)\left(2 \varepsilon_{2}+1\right) / 2 \varepsilon_{2}-\left(\varepsilon_{1}-1\right)\right] \cdot x_{2} \cdot V_{2} / \mathrm{V}$

Donde el volumen molar de la mezcla $V$ es calculado a partir de los volúmenes molares de los solventes $V_{1}$ y $V_{2}$ como:

$V=x_{1} \cdot V_{1}+x_{2} V_{2}$

\subsection{Contribuciones convencionales de UMIFAC:}

La ecuación de UMIFAC consta de dos partes aditivas, un término combinatorio $\gamma_{n}^{c}$ que 
considera el tamaño molecular y las diferencias de forma, y un término residual $\gamma_{n}^{R}$ que considera las interacciones moleculares. La función $\gamma_{n}^{c}$ contiene únicamente parámetros de especie pura, mientras la función $\gamma_{n}^{R}$ incorpora dos parámetros binarios por cada par de moléculas para un sistema multicomponente.

\subsubsection{Parte Combinatoria:}

$1 n \gamma_{n}^{c}=1-j_{n}+1 n j_{n}-5 a_{n}\left(1-\frac{J_{n}}{L_{n}}+1 n \frac{J_{n}}{L_{n}}\right)$

Además se aplican las siguientes funciones:

$$
\begin{array}{lll}
r_{n}=\sum_{k} v_{k}^{(n)} \cdot R_{k} & (9) & a_{n}=\sum_{k} v_{k}^{(n)} \cdot Q_{k} \\
j_{n}=\frac{r_{n}}{\sum_{j} r_{j} \cdot x_{j}} & (11) & L_{n}=\frac{q_{n}}{\sum_{j} a_{j} \cdot x_{j}}
\end{array}
$$

\subsubsection{Parte Residual:}

$\ln \gamma_{n}^{R}=q_{n}\left[1-\sum_{k}\left(\theta_{k} \frac{\beta_{n k}}{S_{k}}-e_{k n} \ln \left(\frac{\beta_{n k}}{S_{k}}\right)\right)\right]$

Además se aplican las siguientes funciones:

$$
\begin{array}{lll}
\tau_{m k}=\exp \left(-\frac{\boldsymbol{a}_{m k}}{T}\right) & (14) & \mathrm{e}_{k n}=\frac{\mathrm{V}_{k}^{n} \cdot Q_{k}}{q_{n}} \\
\theta_{k}=\frac{\sum_{n} x_{n} \cdot q_{n} \cdot e_{k n}}{\sum_{j} X_{j} \cdot q_{j}} \quad(16) & \beta_{n k}=\sum_{m} e_{m n} \cdot \tau_{m k} \\
S_{k}=\sum_{m} \theta_{m} \cdot \tau_{m k} \quad(18) &
\end{array}
$$

El subíndice $n$ identifica las especies y $j$ es un índice ficticio que se ocupa en todas las especies. El subíndice $k$ identifica los grupos y $m$ es un índice ficticio que va en todos los subgrupos. La cantidad $V_{k}^{R}$ es el número de subgrupos del tipo $k$ en una molécula de especie $n$. Los valores de los parámetros de los subgrupos $R_{k}$ y $Q_{k}$ así como los parámetros $a_{m k}$ de interacción entre los grupos solventes e iones resultan de tabulaciones, todos estos son tomados de Kikic et al. (1991). Cuando es posible construir una molécula a partir de más de un conjunto de subgrupos, el conjunto que contiene el menor número de subgrupos diferentes es un conjunto correcto. La gran ventaja del método de UMIFAC es que combina un número relativamente pequeño de subgrupos para formar un número muy grande de moléculas.

\section{DESARROLLO TERMODINÁMICO DEL ELV PARA UN SISTEMA SOLVENTE-SAL}

Para el desarrollo de un equilibrio líquido vapor de un sistema seudobinario alcohol-agua en presencia de electrolito, se debe partir desde el criterio termodinámico fundamental de equilibrio:

$\mathrm{K}_{i}=\frac{\mathrm{Y}_{i}}{x_{i}}=\left(\frac{\mathrm{y}_{i} \mathrm{P}_{i}^{\text {sat }} \mathrm{P}}{\mathrm{P}}\right) \times\left\{\left(\frac{\phi_{i}^{\text {sat }}}{\phi_{i}}\right) \times \exp \left[\frac{\mathrm{V}_{i}^{\text {sat }}}{R T} \times\left(\mathrm{P}-\mathrm{P}_{i}^{\text {sat }}\right)\right]\right\}$

A bajas presiones en los sistemas aquí tratados el factor de corrección de Poynting se acerca de manera considerable a 1 , los coeficientes de fugacidad son calculados a partir de la ecuación de estado Peng-Robinson y se asume además que la sal no está presente en la fase vapor, el coeficiente de actividad es calculado a partir del modelo UMIFAC-E descrito anteriormente, este coeficiente de actividad en sistemas solvente-sal modifica el coeficiente de distribución $K_{i}$ de cada componente en la solución, modificando de manera global el equilibrio líquido vapor. Los sistemas aquí desarrollados para el cálculo del ELV salino fueron los siguientes:

1. Etanol (1)-Agua (2)- $\mathrm{CaCl}_{2}$ (3)

2. 2-Propanol (1)-Agua (2)-LiCl (3)

3. Etanol (1)-Agua (2)-KI (3)

4. Metanol (1)-Agua (2)- $\mathrm{CaCl}_{2}$ (3)

5. 1-Propanol (1)-Agua (2)- $\mathrm{CaCl}_{2}$ (3)

En la Tabla 1 se presentan los parámetros convencionales de volumen y área superficial de UMIFAC, igualmente en la Tabla 2 se muestran los parámetros de interacción $a_{i, j}$ los cuales fueron usados en el desarrollo del presente trabajo. 
Tabla 1. Parámetros de volumen $\left(R_{k}\right)$ y área superficial $\left(Q_{k}\right)$ de UMIFAC

\begin{tabular}{ccccccc}
\hline & \multicolumn{3}{c}{ Cationes } & \multicolumn{3}{c}{ Aniones } \\
\hline Tipo & $\mathrm{Ca}^{+2}$ & $\mathrm{~K}^{+}$ & $\mathrm{Na}^{+}$ & $\mathrm{Li}^{+}$ & $\mathrm{Cl}^{-}$ & $\mathrm{I}^{-}$ \\
$\mathrm{R}_{\mathrm{k}}$ & 1,0 & 3,0 & 3,0 & 1,0 & 0,9861 & 1,6807 \\
$\mathrm{Q}_{\mathrm{k}}$ & 1,0 & 3,0 & 3,0 & 1,0 & 0,9917 & 1,4118 \\
\hline
\end{tabular}

Solventes

\begin{tabular}{cccccc}
\hline $\mathrm{N}_{\mathrm{O}}$ & Grupo principal & $\mathrm{N}_{\mathrm{O}}$ & Subgrupo & $\mathrm{R}_{\mathrm{k}}$ & $\mathrm{Q}_{\mathrm{k}}$ \\
\hline 1 & $\mathrm{CH}_{2}$ & 1 & $\mathrm{CH}_{3}$ & 0,9011 & 0,848 \\
& & 2 & $\mathrm{CH}_{2}$ & 0,6744 & 0,540 \\
& & 3 & $\mathrm{CH}$ & 0,4469 & 0,228 \\
5 & $\mathrm{OH}$ & 15 & $\mathrm{OH}$ & 1,0000 & 1,200 \\
6 & $\mathrm{CH}_{3} \mathrm{OH}$ & 16 & $\mathrm{CH}_{3} \mathrm{OH}$ & 1,4311 & 1,432 \\
7 & $\mathrm{H}_{2} \mathrm{O}$ & 17 & $\mathrm{H}_{2} \mathrm{O}$ & 0,9200 & 1,400 \\
\hline
\end{tabular}

Tabla 2. Parámetros de interacción ai,j en Kelvin

\begin{tabular}{|c|c|c|c|c|c|}
\hline & $\mathrm{CH}_{2}$ & $\mathrm{OH}$ & $\mathrm{CH}_{3} \mathrm{OH}$ & $\mathrm{H}_{2} \mathrm{O}$ & $\mathrm{Ca}^{+2}$ \\
\hline $\mathrm{CH}_{2}$ & 0,0 & 986,5 & 697,2 & 1318,00 & $-433,2$ \\
\hline $\mathrm{OH}$ & 156,4 & 0,0 & $-137,1$ & 353,50 & 5779,7 \\
\hline $\mathrm{CH}_{3} \mathrm{OH}$ & 16,51 & 249,1 & 0,0 & $-181,0$ & $-601,2$ \\
\hline $\mathrm{H}_{2} \mathrm{O}$ & 300,0 & $-229,1$ & 289,6 & 0,0 & $-897,2$ \\
\hline $\mathrm{Ca}^{+2}$ & 955,4 & 3343,7 & $-837,4$ & $-838,2$ & 0,0 \\
\hline $\mathrm{K}^{+}$ & 753,2 & 30,790 & 209,4 & $-248,2$ & n. $c$ \\
\hline $\mathrm{Na}^{+}$ & 1681,1 & 783,6 & 2860,8 & $-165,0$ & n. c \\
\hline $\mathrm{Li}^{+}$ & 4651,5 & $-616,0$ & $-789,23$ & $-612,95$ & n. $c$ \\
\hline $\mathrm{Cl}^{-}$ & 1991,2 & $-367,7$ & 7604,5 & $-230,2$ & 21983 \\
\hline \multirow[t]{2}{*}{$\mathrm{I}^{-}$} & 9183,5 & $-864,67$ & n.d & $-733,4$ & n. d \\
\hline & $\mathrm{K}^{+}$ & $\mathrm{Na}^{+}$ & $\mathrm{Li}^{+}$ & $\mathrm{Cl}^{-}$ & $\mathrm{I}^{-}$ \\
\hline $\mathrm{CH}_{2}$ & 103,7 & 1257,7 & 6434,4 & 68,14 & $-1796,4$ \\
\hline $\mathrm{OH}$ & 87,98 & 1610,4 & $-6,628$ & $-586,4$ & 31892,6 \\
\hline $\mathrm{CH}_{3} \mathrm{OH}$ & 89,21 & $-287,2$ & $-71,347$ & 102,8 & n. $d$ \\
\hline $\mathrm{H}_{2} \mathrm{O}$ & 99,73 & 22,38 & $-404,64$ & $-982,5$ & $-1366,9$ \\
\hline $\mathrm{Ca}^{+2}$ & n. c & n. c & n. $c$ & 4166,3 & n. $d$ \\
\hline $\mathrm{K}^{+}$ & 0,0 & n. c & n. $c$ & 205,9 & $-4359,6$ \\
\hline $\mathrm{Na}^{+}$ & n. c & 0,0 & n. c & 6342,2 & 7535,2 \\
\hline $\mathrm{Li}^{+}$ & n. $c$ & n. $c$ & 0,0 & 22989,1 & n. $d$ \\
\hline $\mathrm{Cl}^{-}$ & $-704,8$ & 14548 & 2795,6 & 0,0 & n. c \\
\hline $\mathrm{I}^{-}$ & 1479,6 & 50016,7 & n. $d$ & n. $c$ & 0,0 \\
\hline
\end{tabular}




\section{CONCLUSIONES Y RESULTADOS}

Para los sistemas descritos anteriormente, se desarrolló el ELV salino, teniendo en cuenta la formulación del equilibrio hecha en la Ecuación (19). En los dos primeros sistemas se calculó el ELV con el fin de comparar el modelo con datos experimentales (Mishi 1975 y Sada et al. 1975), tal como se muestra en las figuras 1 y 2 . De ellas se observa que el modelo UMIFAC-E predice correctamente el efecto de la adición de una sal en un sistema azeotrópico, logrando el desplazamiento total del azeótropo, que a las

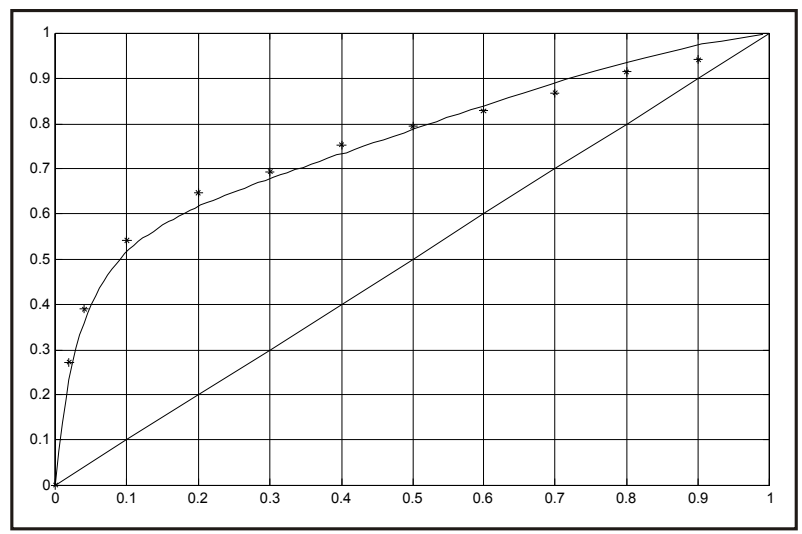

Figura 1. ELV etanol (1)-agua (2) en presencia de $\mathrm{CaCl}_{2}$ al $16.7 \%$ en peso. $P=101.325$ Kpa (-modelo UMIFAC-E, * datos experimentales tomados de Mishi, 1975).

La fracción molar de la fase líquida está expresada en fracción libre de solvente. condiciones termodinámicas planteadas en las figuras, corresponden a una fracción molar de etanol de 0.8933 en la Figura 1 y 0.6871 de 2 propanol en la Figura 2.

Adicional a la comparación gráfica, en la Tabla 3 se muestran las desviaciones de la fracción molar de la fase vapor con respecto a los datos experimentales para los sistemas ternarios Etanol-Agua-CaCl ${ }_{2}$ y 2-propanol-Agua-LiCl. Así mismo, dichas desviaciones se comparan con los valores reportados para los mismos sistemas por Chen et al. (1986).

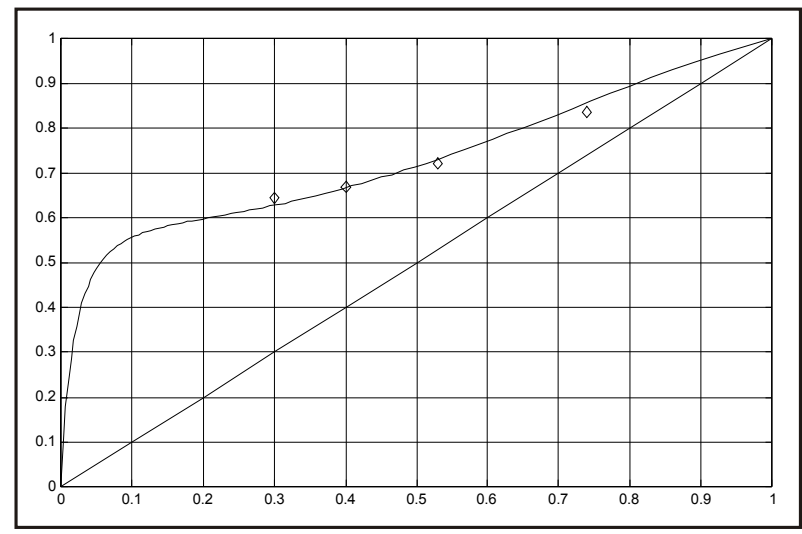

Figura. 2 ELV 2-propanol-agua en presencia de cloruro de litio. T=348 K. (-modelo UMIFAC-E con 10\% peso de LiCl, datos experimentales tomados de Sada et al., 1975).

Tabla 3. Desviación promedio de la fracción molar vapor de alcohol

\begin{tabular}{cccc}
\hline & \multicolumn{2}{c}{ Etanol-Agua-CaCl 2} \\
\hline Concentración sal & NRTL & UNIFAC-E & Referencia datos \\
\%peso & Chen et al., 1986 & Este trabajo & experimentales \\
16.7 & 0.024 & 0.025 & Nishi (1975) \\
\hline \multicolumn{4}{c}{ 2-propanol-Agua-LiCl } \\
Concentración sal & NRTL & UNIFAC-E & Referencia datos \\
Saturada & Chen et al., 1986 & Este trabajo & experimentales \\
& 0.006 & 0.012 & Sada et al. (1975) \\
\hline
\end{tabular}


Los valores de desviación promedio para la fase vapor mostrados en la Tabla 3, expresan la buena reproducción de los equilibrios que tiene el modelo UMIFAC-E, lo que lo convierte en una muy buena herramienta a la hora de diseñar equipos de separación de contacto líquido-vapor que involucren sistemas electrolíticos. En la Figura 3 puede observarse el grado de desplazamiento de la curva de equilibrio en el sistema etanol (1)agua (2) en presencia de cloruro de calcio con una concentración molal de sal de $1.68 \mathrm{~m}$, donde se compara con el sistema en ausencia de sal, por lo que se logra demostrar la eficacia de la adición de la sal en este sistema azeotrópico. Una característica del cloruro de calcio es que posee un ión divalente: $\mathrm{Ca}^{+2}$, la particularidad de este tipo de sales es que son más eficientes que las sales monovalentes a la hora de alejar la curva de equilibrio de la línea de $45^{\circ}$, debido a la fuerza iónica de sus iones disueltos en la mezcla de solventes, este efecto es mostrado en la Figura 4,

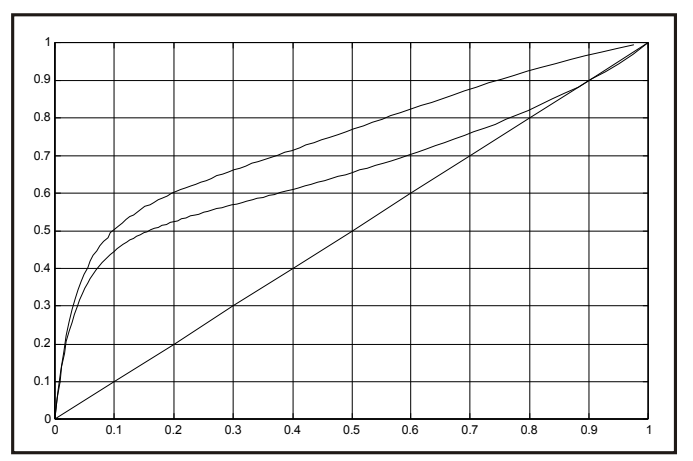

Figura 3. ELV etanol (1)-agua (2). $P=100 \mathrm{Kpa}$ (- ELV con $1.68 \mathrm{~m}$ de $\mathrm{CaCl}_{2}$,-- --ELV libre de sal).

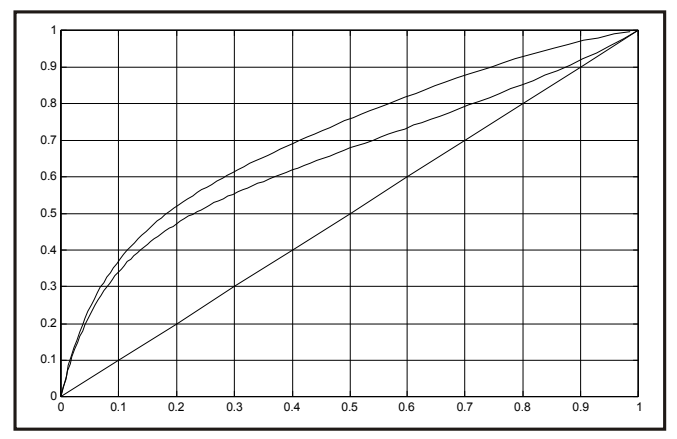

Figura 5. ELV metanol (1)-agua (2). P=101.325 Kpa con $11.5 \%$ en peso de sal. (- - en ausencia de sal, ---- $\mathrm{CaCl}_{2}$ ). donde se hace una comparación del efecto salino de una sal monovalente (KI) con una sal divalente $\left(\mathrm{CaCl}_{2}\right)$, a una misma concentración de sal.

En el desarrollo del ELV de un sistema de solventes zeotrópicos, caso similar al de solventes azeotrópicos, la adición de una sal modifica el equilibrio en la forma deseada: alejamiento de la curva de equilibrio de la línea de $45^{\circ}$, por lo cual, al momento de diseñar una columna de destilación extractiva por efecto salino se presentará una disminución beneficiosa en el número de etapas de la torre. En la Figura 5 se muestra este caso, para el sistema metanolagua con adición de $\mathrm{CaCl}_{2}$. En el sistema 1propanol-agua, la formulación del equilibrio en presencia de sal lleva a lo mostrado en la Figura. 6 , donde se logra observar el desplazamiento total del azeótropo y el alejamiento de la curva de equilibrio de la línea de $45^{\circ}$ gracias a la adición de la sal.

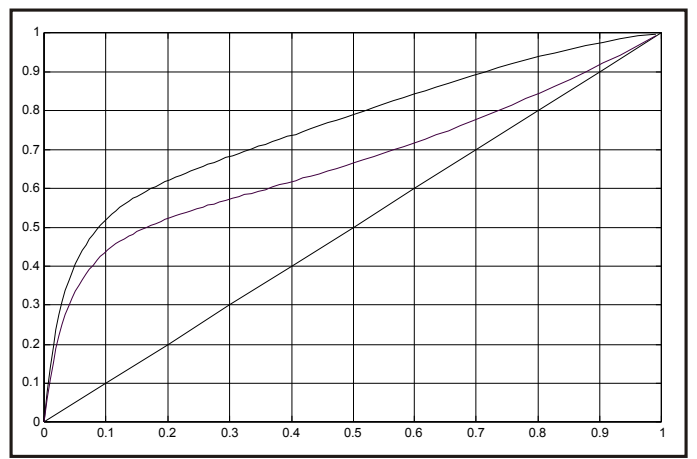

Figura 4. ELV etanol (1)-agua (2). Comparación del efecto salino. $P=78 \mathrm{Kpa}$ con $16.7 \%$ en peso de sal. (- $\mathrm{Kl}$, -- -- $\mathrm{CaCl}_{2}$ ).

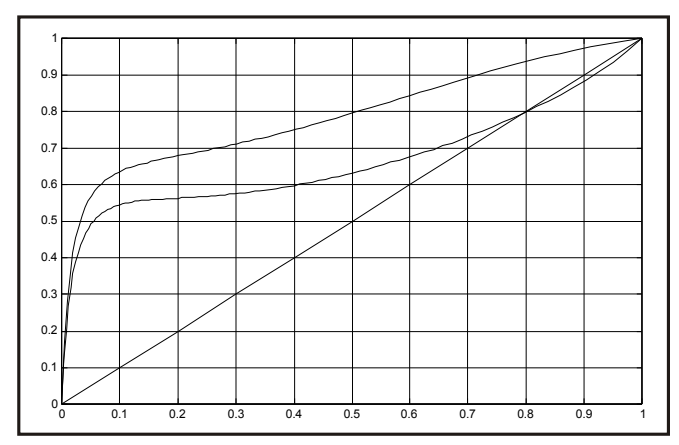

Figura 6. ELV 1-propanol (1)-agua (2). P=78 Kpa con $18 \%$ en peso de sal. (- - - en ausencia de sal, --- $\left(\mathrm{CaCl}_{2}\right)$.

Volumen 6 - No. 2 - Diciembre de 2004 
El efecto que sobre el coeficiente de actividad de un solvente en solución tiene la adición de una sal en los sistemas alcohol-agua aquí mostrados es favorable a la hora del diseño técnico de un equipo de separación, los beneficios son altos: reducción de costos en el agente separador comparado con operaciones similares que usan agentes orgánicos, disminución sustancial de los costos energéticos de operación de la torre debido a que al adicionar la sal en la mezcla, ésta aumenta las volatilidades en el rehervidor, disminuyendo la carga térmica en el mismo. Adicional a dichas ventajas y gracias al efecto salino en el desplazamiento del azeótropo y el alejamiento de la curva de equilibrio de la línea de $45^{\circ}$, se obtiene además una alta pureza de producto, que en muchos de los casos alcanza niveles mayores a los obtenidos en operaciones de separación más costosas.

\section{REFERENCIAS}

$\square$ Abbott. M. M, Prausnitz. J. M. Models for Thermodynamic and phase equilibria calculations. Marcel Dekker, Inc. (1994).

च Cardoso. M. \& O'Conell. J. P. Activity coefficients in mixed solvent electrolyte solutions. Fluid Phase Equilibria 33, 315. (1987).

घ Chen. C. C., Evans. L. B. \& Mock. B. Thermodynamic representation of phase equilibria of mixed-solvent electrolyte systems. American Institute of Chemical Engineers journal 32, 1655-1664. (1986).

घ Kikic, I., Fermeglia, M., \& Rasmussen, P. UMIFAC prediction of vapor-liquid equilibria in mixed solvent-salt systems. Chemical Engineering Science 46, 2775-2780. (1991)

■ Macedo, E. A., Skovborg, P., \& Rasmussen, P. Calculation of phase equilibria for solutions of strong electrolytes in solvent-water mixtures. Chemical Engineering Science 45, 875-882. $\square$ Mishi, Y. Vapor-liquid equilibrium relations for the system accompanied by hypothetical chemical reaction containing salt. Journal of Chemical Engineering of Japan 8, 187-191. (1975).

ఐ Sada, E., Morisue. T., \& Miyahara. K. Salt effects on Vapor-Liquid Equilibrium of isopropanol-water systems. Journal of Chemical Engineering of Japan 8(3), 196. (1975).

■ Sander, B., Fredenslund, A., \& Rasmussen, P. Calculation of vapor-liquid equilibria in mixed solvent/salt systems using an extended UNIQUAC equation. Chemical Engineering Science 41, 1171-1183. (1986).

$\square$ Zhao. E., Yu. M., Sauvé. R. E. \& Khoshkbarchi. M. K. Extension of the Wilson model to electrolyte solutions. Fluid Phase Equilibria 173, 161-175. (2000). 


\section{NOMENCLATURA}

\begin{tabular}{|c|c|}
\hline A & Parámetro de Debye-Hückel \\
\hline $\mathrm{a}_{\mathrm{mk}}$ & Parámetros de interacción entre los grupos de solventes e iones \\
\hline $\mathrm{b}$ & Parámetro de Debye-Hückel \\
\hline$d_{n}$ & Densidad del solvente $n$ \\
\hline $\mathrm{d}_{\mathrm{S}}$ & Densidad de la mezcla de solventes \\
\hline I & Fuerza iónica \\
\hline $\mathrm{K}_{\mathrm{i}}$ & Coeficiente de distribución del componente i \\
\hline $\mathrm{M}_{\mathrm{n}}$ & Masa molar del solvente $\mathrm{n}$ \\
\hline $\mathrm{M}_{\mathrm{S}}$ & Masa molar de la mezcla líquida \\
\hline $\mathrm{P}$ & Presión total del sistema \\
\hline$P_{i}^{s a t}$ & Presión de saturación del solvente $i$ \\
\hline $\mathrm{Q}_{\mathrm{k}}$ & Parámetros de los subgrupos solventes e iones de ELV-UMIFAC \\
\hline $\mathrm{R}$ & Constante universal de los gases \\
\hline $\mathrm{R}_{\mathrm{k}}$ & Parámetros de los subgrupos solventes e iones de ELV-UMIFAC \\
\hline $\mathrm{T}$ & Temperatura absoluta del sistema \\
\hline $\mathrm{V}$ & Volumen molar de la mezcla \\
\hline$v_{i}^{\text {sat }}$ & Volumen de líquido saturado \\
\hline$v_{k}^{n}$ & ero de subgrupos de tipo k en una molécula de la especie $n$. \\
\hline $\mathrm{V}_{1}, \mathrm{~V}_{2}$ & Volúmenes molares de los solventes 1 y 2 , respectivamente. \\
\hline$x_{n}^{\prime}$ & Fracción molar del solvente n libre de sal \\
\hline $\mathrm{X}_{\mathrm{i}}$ & Fracción molar de la fase líquida del componente i \\
\hline$y_{i}$ & Fracción molar de la fase vapor del componente i \\
\hline \multicolumn{2}{|c|}{ Letras griegas } \\
\hline$\gamma_{\mathrm{n}}$ & Coeficiente de actividad del solvente $n$ \\
\hline$\gamma_{n}^{C}$ & Coeficiente de actividad combinatorio del solvente $n$ \\
\hline$\gamma_{n}^{D-H}$ & Coeficiente de actividad de Debye-Hückel para solvente $n$ \\
\hline$\gamma_{n}^{R}$ & Coeficiente de actividad residual del solvente $\mathrm{n}$ \\
\hline$\phi_{i}^{\text {sat }}$ & saturado del solvente $i$ \\
\hline$\varepsilon$ & éctrica de la mezcla solventes \\
\hline & rica del solvente puro \\
\hline
\end{tabular}

